\title{
Research Article \\ Effects of Thermocapillarity and Thermal Radiation on Flow and Heat Transfer in a Thin Liquid Film on an Unsteady Stretching Sheet
}

\author{
R. C. Aziz, ${ }^{1}$ I. Hashim, ${ }^{2}$ and S. Abbasbandy ${ }^{3}$ \\ ${ }^{1}$ Faculty of Science and Technology, Open University Malaysia, 50480 Kuala Lumpur, Malaysia \\ ${ }^{2}$ Solar Energy Research Institute, Universiti Kebangsaan Malaysia, Selangor, 43600 Bangi, Malaysia \\ ${ }^{3}$ Department of Mathematics, Imam Khomeini International University, Ghazvin 34149-16818, Iran \\ Correspondence should be addressed to I. Hashim, ishak_h@ukm.my
}

Received 17 August 2011; Revised 20 October 2011; Accepted 26 October 2011

Academic Editor: Ben T. Nohara

Copyright (c) 2012 R. C. Aziz et al. This is an open access article distributed under the Creative Commons Attribution License, which permits unrestricted use, distribution, and reproduction in any medium, provided the original work is properly cited.

This paper examines the effects of thermocapillarity and thermal radiation on the boundary layer flow and heat transfer in a thin film on an unsteady stretching sheet with nonuniform heat source/ sink. The governing partial differential equations are converted into ordinary differential equations by a similarity transformation and then are solved by using the homotopy analysis method (HAM). The effects of the radiation parameter, the thermocapillarity number, and the temperaturedependent parameter in this study are discussed and presented graphically via velocity and temperature profiles.

\section{Introduction}

The analysis of heat transfer of boundary layer flow with thermal radiation is important in electrical power generation, astrophysical flows, solar power technology, space vehicle reentry, and other industrial engineering processes.

Wang [1] was the pioneer in investigating the hydrodynamics of a flow in a thin liquid film on an unsteady stretching surface. Later, Andersson et al. [2] studied the heat transfer characteristics of the hydrodynamical problem solved by Wang [1]. Liu and Andersson [3] examined the problem with a more general form of prescribed temperature variation of a stretching sheet. Wang [4] investigated the same problem of Andersson et al. [2], presenting analytic solutions. Several researchers have extended Wang's [1] classical problem, taking into consideration non-Newtonian [5-9], thermocapillarity [10-13], and magnetic effects [14, $15]$. 
Wang [4] was probably the first to analyze the flow and heat transfer in a thin liquid film on an unsteady stretching surface using the homotopy analysis method (HAM). The HAM is a general analytic method for obtaining series solutions for various types of nonlinear equations [16]. Several studies have successfully applied HAM to various nonlinear problems in science and engineering [17-22]. Studies by Dandapat et al. [10,11] reported that thermocapillarity generates surface-tension gradients along the horizontal interface between the passive gas and the liquid film. Both Abd El-Aziz [23] and Mahmoud [24] have studied the influence of thermal radiation on the flow and heat transfer on an unsteady stretching sheet.

Motivated by these studies, in this paper we extend the results of Wang [4] and Mahmoud [24] for thin film flow on an unsteady stretching sheet with combined effects of thermocapillarity, thermal radiation, and internal heating. Furthermore, we employ the similarity transformation introduced by Wang [4] to transform the extent of the independent variable into a finite range $0-1$. The solutions reached using HAM are presented and implications discussed.

\section{Problem Formulation}

The fluid flow, modeled as an unsteady, two-dimensional, incompressible viscous laminar flow on a horizontal thin elastic sheet, emerges from a narrow slot at the origin of a Cartesian coordinate system. Fluid motion and heat transfer arise in the stretching of the horizontal elastic sheet. It is assumed that the elastic sheet has internal heat generation/absorption and that the sheet temperature varies with the coordinate $x$ and time $t$. Under these assumptions, the governing conservation equations of mass, momentum, and energy at unsteady state can be expressed as

$$
\begin{gathered}
\frac{\partial u}{\partial x}+\frac{\partial v}{\partial y}=0 \\
\frac{\partial u}{\partial t}+u \frac{\partial u}{\partial x}+v \frac{\partial u}{\partial y}=v \frac{\partial^{2} u}{\partial y^{2}} \\
\rho C_{p}\left(\frac{\partial T}{\partial t}+u \frac{\partial T}{\partial x}+v \frac{\partial T}{\partial y}\right)=\kappa \frac{\partial^{2} T}{\partial y^{2}}+Q-\frac{\partial q_{r}}{\partial y},
\end{gathered}
$$

subject to

$$
\begin{gathered}
u=U, \quad v=0, \quad T=T_{s} \quad \text { at } y=0, \\
\mu \frac{\partial u}{\partial y}=\frac{\partial \sigma}{\partial x}, \quad \frac{\partial T}{\partial y}=0, \quad v=\frac{\mathrm{d} h}{\mathrm{~d} t} \quad \text { at } y=h,
\end{gathered}
$$

where $u$ and $v$ are the velocity components of the fluid in the $x$-and $y$-directions, $t$ is the time, $T$ is the temperature, $v$ is the kinematic viscosity, $\rho$ is the density, $\kappa$ is the thermal diffusivity, $C_{p}$ is the specific heat at constant pressure, $\mu$ is the viscosity, and $h(t)$ is the uniform thickness of the liquid film. Note that the surface tension varies linearly with temperature, defined as $\sigma=\sigma_{0}\left[1-\delta\left(T-T_{0}\right)\right]$, where $\delta$ is a positive fluid property. The velocity of the stretching surface 
is defined as $U=b x /(1-\alpha t)$, with $\alpha$ and $b$ as positive constants. The $Q$ term is the heat generated $(>0)$ or absorbed $(<0)$ per unit volume and is defined as

$$
Q=\left(\frac{\kappa U}{x \mathcal{v}}\right) B^{*}\left(T-T_{o}\right)
$$

where $B^{*}$ is the temperature-dependent heat generation/absorption, that is, $B^{*}$ is positive in the case of the elastic sheet's generation of heat and negative in the case of the sheet's absorption of heat from the fluid flow. The radiative heat flux $q_{r}$ under Rosseland approximation (Brewster, 1992, cited in [23]) is given in the form

$$
q_{r}=-\frac{4 \sigma_{*}}{3 k_{1}} \frac{\partial T^{4}}{\partial y}
$$

where $\sigma_{*}$ is the Stefan Boltzmann constant and $k_{1}$ is the mean absorption coefficient. We assume that the temperature difference within the flow is sufficiently small for $T^{4}$ to be expressed as a linear function of temperature. This is accomplished by expanding $T^{4}$ in a Taylor series about $T_{0}$ and neglecting higher-order terms, thus

$$
T^{4} \cong 4 T_{0}^{3} T-3 T_{0}^{4}
$$

According to Wang [4], the surface temperature $T_{S}$ of the stretching sheet varies with the distance $x$ from the slot and time $t$ in the form

$$
T_{s}=T_{o}-T_{\text {ref }} \frac{b x^{2}}{2 v}(1-\alpha t)^{-3 / 2},
$$

where $T_{o}$ is the temperature at the slit, $T_{\text {ref }}$ is the constant reference temperature for all $t<1 / \alpha$. The surface of the planar liquid film is assumed to be smooth and free of surface waves while viscous shear stress and heat flux are assumed to vanish at the adiabatic free surface. Liu and Andersson [3] developed similarity transformations, which are given as

$$
\begin{gathered}
\psi=\beta x\left[\frac{v b}{1-\alpha t}\right]^{1 / 2} f(\eta), \\
T=T_{o}-T_{\operatorname{ref}}\left[\frac{d x^{2}}{v(1-\alpha t)^{3 / 2}}\right] \theta(\eta), \\
\eta=\frac{1}{\beta}\left[\frac{b}{v(1-\alpha t)}\right]^{1 / 2} y,
\end{gathered}
$$

where $\beta$ is the dimensionless film thickness and $\psi(x, y)$ is the stream function defined by

$$
\begin{gathered}
u=\frac{\partial \psi}{\partial y}=\frac{b x}{1-\alpha t} f^{\prime}(\eta), \\
v=-\frac{\partial \psi}{\partial x}=-\left(\frac{v b}{1-\alpha t}\right)^{1 / 2} \beta f(\eta),
\end{gathered}
$$


where a prime denotes differentiation with respect to $\eta$. Apparently, (2.10) have already satisfied (2.1). Consequently, (2.1)-(2.4) are transformed to the following nonlinear boundary value problem:

$$
\begin{gathered}
f^{\prime \prime \prime}+r\left(f f^{\prime \prime}-\frac{1}{2} S \eta f^{\prime \prime}-\left(f^{\prime}\right)^{2}-S f^{\prime}\right)=0 \\
\frac{1}{\operatorname{Pr}}\left(1+N_{R}\right) \theta^{\prime \prime}+r\left(f \theta^{\prime}-2 f^{\prime} \theta-\frac{1}{2} S \eta \theta^{\prime}-\frac{3}{2} S \theta+\frac{1}{\operatorname{Pr}} B^{*} \theta\right)=0
\end{gathered}
$$

subject to

$$
\begin{gathered}
f(0)=0, \quad f^{\prime}(0)=1, \quad \theta(0)=1, \\
f(1)=\frac{1}{2} S, \quad f^{\prime \prime}(1)=M \theta(1), \quad \theta^{\prime}(1)=0,
\end{gathered}
$$

where a prime denoting differentiation with respect to $\eta, S=\alpha / 2$ is the dimensionless measure of unsteadiness, Pr is the Prandtl number, $N_{R}$ is the radiation parameter defined as $N_{R}=$ $16 \sigma_{*} T_{0}^{3} / \kappa k_{1}$, and $\gamma=\beta^{2}$ is an unknown constant which must be determined as a part of the present problem. The thermocapillarity number $M$ is given as

$$
M=\frac{\beta \delta \sigma_{0} T_{\mathrm{ref}}}{\mu \sqrt{b v}} .
$$

It is worth noting that the momentum boundary layer problem defined by (2.11) subject to the relevant boundary conditions (2.13) is decoupled from the thermal boundary layer problem, while the temperature field $\theta(\eta)$ is on the other hand coupled to the velocity field. The most important characteristics of flow and heat transfer are shear stress $\tau_{s}$ and heat flux $q_{s}$ of the stretching sheet, which are defined as

$$
\begin{gathered}
\tau_{s}=\mu\left(\frac{\partial u}{\partial y}\right)_{y=0}, \\
q_{s}=-\kappa\left(\frac{\partial T}{\partial y}\right)_{y=0},
\end{gathered}
$$

where $\mu$ is the fluid dynamic viscosity. The local skin-friction coefficient $C_{f}$ and the local Nusselt number $\mathrm{Nu}_{x}$ can be defined as

$$
\begin{gathered}
C_{f}=\frac{2 \tau_{s}}{\rho u^{2}}, \\
\mathrm{Nu}_{x}=-\frac{x q_{s}}{\kappa T_{\text {ref }}} .
\end{gathered}
$$


Thus, the skin friction and the rate of heat transfer for fluid flow in a thin film can be expressed as

$$
\begin{gathered}
\frac{1}{2} C_{f} \operatorname{Re}_{x}^{1 / 2}=\frac{1}{\beta} f^{\prime \prime}(0), \\
\mathrm{Nu}_{x} \operatorname{Re}_{x}^{-1 / 2}=\frac{d x^{2}}{\beta v(1-\alpha t)^{1 / 2}} \theta^{\prime}(0),
\end{gathered}
$$

where $\operatorname{Re}_{x}=U x / v$ is the local Reynolds number.

\section{Solution Approach}

In this section we apply HAM to solve system (2.11)-(2.14). We assume that the solutions of $f(\eta)$ and $\theta(\eta)$ can be expressed by a set of base functions $\left\{\eta^{m} \mid m=0,1,2, \ldots\right\}$ with the following forms:

$$
\begin{aligned}
& f(\eta)=\sum_{m=0}^{+\infty} a_{m} \eta^{m} \\
& \theta(\eta)=\sum_{m=0}^{+\infty} c_{m} \eta^{m}
\end{aligned}
$$

where $a_{m}$ and $c_{m}$ are constants. Under the rule of solution expression given by (2.11) and (2.12), subject to the boundary conditions (2.13) and (2.14), it is straightforward to choose

$$
\begin{gathered}
f_{0}(\eta)=\eta+\frac{3 S-6-M \theta_{0}(\eta)}{4} \eta^{2}+\frac{2-S+M \theta_{0}(\eta)}{4} \eta^{3}, \\
\theta_{0}(\eta)=1
\end{gathered}
$$

as the initial guesses of $f(\eta)$ and $\theta(\eta)$. The auxiliary linear operators $L_{f}=\partial^{3} / \partial \eta^{3}$ and $L_{\theta}=$ $\partial^{2} / \partial \eta^{2}$ are chosen with the following properties:

$$
\begin{gathered}
L_{f}\left[C_{1}+C_{2} \eta+C_{3} \eta^{2}\right]=0, \\
L_{\theta}\left[C_{4}+C_{5} \eta\right]=0
\end{gathered}
$$

where $C_{1}, C_{2}, C_{3}, C_{4}$, and $C_{5}$ are constants of integration. From (2.11) and (2.12), the nonlinear operators are defined as

$$
\begin{gathered}
N_{f}[F(\eta, q), \Gamma(q)]=F^{\prime \prime \prime}+\Gamma\left[F F^{\prime \prime}-\frac{1}{2} S \eta F^{\prime \prime}-\left(F^{\prime}\right)^{2}-S F^{\prime}\right] \\
N_{\theta}[F(\eta, q), \Theta(\eta, q), \Gamma(q)]=\frac{1}{\operatorname{Pr}}\left(1+N_{R}\right) \Theta^{\prime \prime}+\Gamma\left[F \Theta^{\prime}-2 F^{\prime} \Theta-\frac{1}{2} S \eta \Theta^{\prime}-\frac{3}{2} S \Theta+\frac{1}{\operatorname{Pr}} B^{*} \Theta\right]
\end{gathered}
$$


where $F(\eta, q)$ and $\Theta(\eta, q)$ are both unknown functions of $\eta$ and $q$, while $\Gamma$ is a function dependent on $q$. The prime denotes differentiation with respect to $\eta$. Let $\hbar_{f}$ and $\hbar_{\theta}$ denote the nonzero auxiliary parameters, whereas $H_{f}$ and $H_{\theta}$ denote nonzero auxiliary functions. The zero-order deformation equation can be constructed as

$$
\begin{gathered}
(1-q) L_{f}\left[F(\eta, q)-f_{0}(\eta)\right]=q \hbar_{f} H_{f} N_{f}[F(\eta, q), \Gamma(q)], \\
(1-q) L_{\theta}\left[\Theta(\eta, q)-\theta_{0}(\eta)\right]=q \hbar_{\theta} H_{\theta} N_{\theta}[F(\eta, q), \Theta(\eta, q), \Gamma(q)],
\end{gathered}
$$

subject to the boundary conditions

$$
\begin{gathered}
F(0, q)=0, \quad F^{\prime}(0, q)=1, \quad \Theta(0, q)=1, \\
F(1, q)=\frac{1}{2} S, \quad F^{\prime \prime}(1, q)=M \theta(1), \quad \Theta^{\prime}(1, q)=0,
\end{gathered}
$$

where $q$ is an embedding parameter. From (3.2), it is straightforward to show that when $q=0$, the solutions of (3.5) and (3.6) are

$$
F(\eta, 0)=f_{0}(\eta), \quad \Theta(\eta, 0)=\theta_{0}(\eta)
$$

Since $\hbar_{f}, \hbar_{\theta} \neq 0$ and $H_{f}, H_{\theta} \neq 0$ when $q=1$, (3.5) and (3.6) are equivalent to (2.11)-(2.14), respectively, provided that

$$
F(\eta, 1)=f(\eta), \quad \Theta(\eta, 1)=\theta(\eta), \quad \Gamma(1)=\gamma .
$$

Thus, as $q$ increases from 0 to $1, F(\eta, q)$ and $\Theta(\eta, q)$ vary from the initial guesses $f_{0}(\eta)$ and $\theta_{0}(\eta)$ to the solutions $f(\eta)$ and $\theta(\eta)$ in (2.11)-(2.14). $\Gamma$ also varies from the initial guess

$$
\Gamma(0)=\gamma_{0},
$$

to the time-scale parameter $\gamma$. Applying the Taylor series to (3.7) and (3.9), $F(\eta, q), \Theta(\eta, q)$, and $\Gamma(q)$ can be expanded as series of $q$,

$$
\begin{gathered}
F(\eta, q)=f_{0}(\eta)+\sum_{m=1}^{+\infty} f_{m}(\eta) q^{m} \\
\Theta(\eta, q)=\Theta_{0}(\eta)+\sum_{m=1}^{+\infty} \theta_{m}(\eta) q^{m}, \\
\Gamma(q)=\gamma_{0}+\sum_{m=1}^{+\infty} \gamma_{m} q^{m},
\end{gathered}
$$


where

$$
\begin{aligned}
f_{m}(\eta) & =\frac{1}{m !}\left[\frac{\partial^{m} F(\eta, q)}{\partial q^{m}}\right]_{q=0}, \\
\theta_{m}(\eta) & =\frac{1}{m !}\left[\frac{\partial^{m} \Theta(\eta, q)}{\partial q^{m}}\right]_{q=0}, \\
\gamma_{m} & =\frac{1}{m !}\left[\frac{\partial^{m} \Gamma(q)}{\partial q^{m}}\right]_{q=0} .
\end{aligned}
$$

Thus, using (3.8), we have

$$
\begin{gathered}
f(\eta)=f_{0}(\eta)+\sum_{m=1}^{+\infty} f_{m}(\eta), \\
\theta(\eta)=\theta_{0}(\eta)+\sum_{m=1}^{+\infty} \theta_{m}(\eta), \\
r=\gamma_{0}+\sum_{m=1}^{+\infty} \gamma_{m} .
\end{gathered}
$$

By differentiating (3.5) $m$ times with respect to $q$, then setting $q=0$, and finally dividing by $m !$, the $m$ th-order deformation equations are obtained:

$$
\begin{aligned}
& L_{f}\left[f_{m}(\eta)-x_{m} f_{m-1}(\eta)\right]=\hbar_{f} H_{f}(\eta) R_{1, m}(\eta), \\
& L_{\theta}\left[\theta_{m}(\eta)-\chi_{m} \theta_{m-1}(\eta)\right]=\hbar_{\theta} H_{\theta}(\eta) R_{2, m}(\eta),
\end{aligned}
$$

subject to the boundary conditions

$$
\begin{gathered}
f_{m}(0)=0, \quad f_{m}^{\prime}(0)=0, \quad \theta_{m}(0)=0, \\
f_{m}(1)=0, \quad f_{m}^{\prime \prime}(1)=M \theta_{m}(1), \quad \theta_{m}^{\prime}(1)=0,
\end{gathered}
$$

for $m \geq 1$, where

$$
\begin{aligned}
R_{1, m}(\eta)= & f_{m-1}^{\prime \prime \prime}+\sum_{n=0}^{m-1} \gamma_{m-1-n} \sum_{i=0}^{n}\left(f_{i} f_{n-i}^{\prime \prime}-f_{i}^{\prime} f_{n-i}^{\prime}\right)-\frac{1}{2} S \eta \sum_{n=0}^{m-1} \gamma_{n} f_{m-1-n}^{\prime \prime}-S \sum_{n=0}^{m-1} \gamma_{n} f_{m-1-n}^{\prime} \\
R_{2, m}(\eta)= & \frac{1}{\operatorname{Pr}} \theta_{m-1}^{\prime \prime}+\frac{N_{R}}{\operatorname{Pr}} \theta_{m-1}^{\prime \prime}+\sum_{n=0}^{m-1} \gamma_{m-1-n} \sum_{i=0}^{n}\left(f_{i} \theta_{n-i}^{\prime}-2 f_{n-i}^{\prime} \theta_{i}\right) \\
& -\frac{1}{2} S \eta \sum_{n=0}^{m-1} \gamma_{n} \theta_{m-1-n}^{\prime}-\frac{3}{2} S \sum_{n=0}^{m-1} \gamma_{n} \theta_{m-1-n}+\frac{1}{\operatorname{Pr}} B^{*} \sum_{n=0}^{m-1} \gamma_{n} \theta_{m-1-n}, \\
X_{m}= & \begin{cases}1 & m>1 \\
0 & m=1 .\end{cases}
\end{aligned}
$$


Let $f_{m}^{*}$ and $\theta_{m}^{*}$ denote the particular solutions of (3.13) as

$$
\begin{gathered}
f_{m}^{*}=\iiint_{0}^{\eta} \hbar_{f} H_{f}(s) R_{1, m}(s) \mathrm{d} s \mathrm{~d} \eta \mathrm{d} \eta+\chi_{m} f_{m-1}, \\
\theta_{m}^{*}=\iint_{0}^{\eta} \hbar_{\theta} H_{\theta}(s) R_{2, m}(s) \mathrm{d} s \mathrm{~d} \eta+\chi_{m} \theta_{m-1} .
\end{gathered}
$$

The general solutions for (3.3) are

$$
\begin{gathered}
f_{m}(\eta)=f_{m}^{*}+C_{1}+C_{2} \eta+C_{3} \eta^{2} \\
\theta_{m}(\eta)=\theta_{m}^{*}+C_{4}+C_{5} \eta
\end{gathered}
$$

It should be pointed out that $f_{m}^{*}$ and $\theta_{m}^{*}$ contain the unknown parameter $\gamma_{m-1}$, which should be determined along with $C_{1}, C_{2}, C_{3}, C_{4}$, and $C_{5}$ by the boundary conditions (3.14). We solve (3.13) for $m=1,2,3, \ldots$ successfully, and the $m$ th-order approximations of $f(\eta), \theta(\eta)$, and $\gamma$ are given, respectively, by

$$
\begin{aligned}
f(\eta) & \approx \sum_{n=0}^{m} f_{n}(\eta), \\
\theta(\eta) & \approx \sum_{n=0}^{m} \theta_{n}(\eta), \\
r & \approx \sum_{n=0}^{m-1} r_{n} .
\end{aligned}
$$

\section{Convergence of the HAM Solution}

We note the presence of the auxiliary parameters $\hbar_{f}$ and $\hbar_{\theta}$ in (3.13). Liao [16, 25] indicates that the convergence and rate of approximation of such series depend on the values of $\hbar_{f}$ and $\hbar_{\theta}$. For the purpose of choosing the proper values for $\hbar_{f}$ and $\hbar_{\theta}$, we set $S=1.4, \operatorname{Pr}=1, M=1$, $N_{R}=1$, and $B^{*}=0.05$. Figure 1 (a) shows the variation of $\gamma$ with $\hbar_{f}$ using the tenth-order approximation. From the figure, it is seen that convergent result can be obtained when $-0.8 \leq$ $\hbar_{f} \leq-0.35$. Thus, we can choose an appropriate value for $\hbar_{f}$ in this range to get convergent solution for $\gamma$. In Figure 1(b), we observe that convergent results of $f^{\prime \prime}(0)$ can be obtained by choosing a value of $\hbar_{f}$ in the range of $-0.78 \leq \hbar_{f} \leq-0.16$ under the tenth-order approximation. From (2.13), we can see that $\hbar_{\theta}$ depends on $\hbar_{f}$. Figure 1 (c) shows the appropriate value for $\hbar_{\theta}$ in the range of $-0.75 \leq \hbar_{\theta} \leq-0.25$ when $\hbar_{f}=-0.6$ under the tenth-order approximation. To see the accuracy of the solution, we define $E f(\eta)$ and $E \theta(\eta)$ as the residual errors of the system. Figure 2 shows the residual errors of the HAM solution in the case $\hbar_{f}=-0.6$ and $\hbar_{\theta}=-0.4$.

\section{Results and Discussion}

We solved (3.13) and (3.14) with the aid of Maple, a symbolic computation software. The auxiliary functions $H_{f}$ and $H_{\theta}$ in (3.13) were set to be equal to 1 in all calculations reported in this paper. 


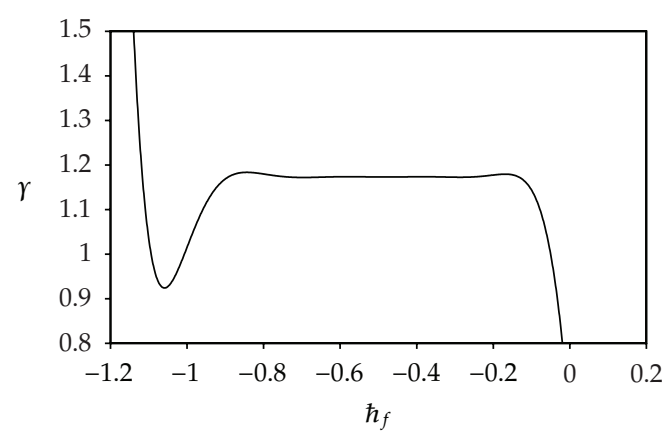

(a)

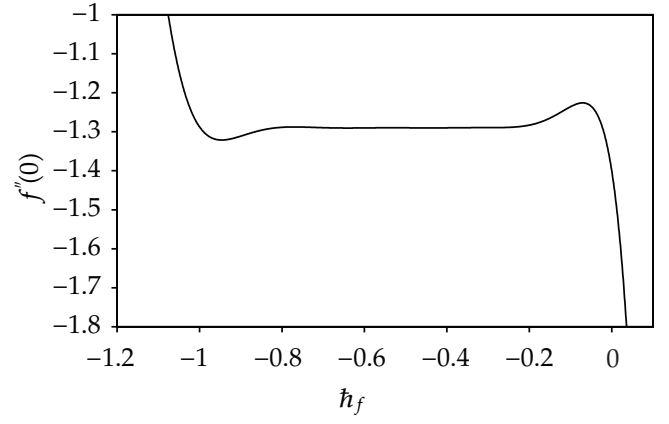

(b)

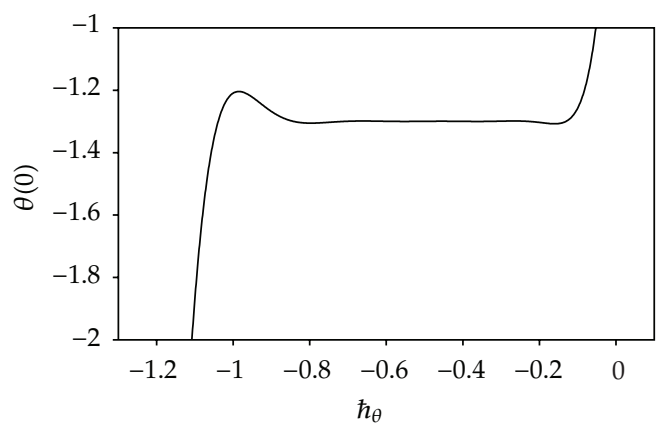

(c)

Figure 1: (a) $\hbar_{f}$-curve of $f^{\prime \prime}(0)$, (b) $\hbar_{f}$-curve of $\gamma$, and (c) $\hbar_{\theta}$-curve of $\theta^{\prime}(0)\left(\hbar_{f}=-0.6\right)$ using 10th-order HAM approximation for the case of $S=1.4, \operatorname{Pr}=1, M=1, N_{R}=1$, and $B^{*}=0.05$.

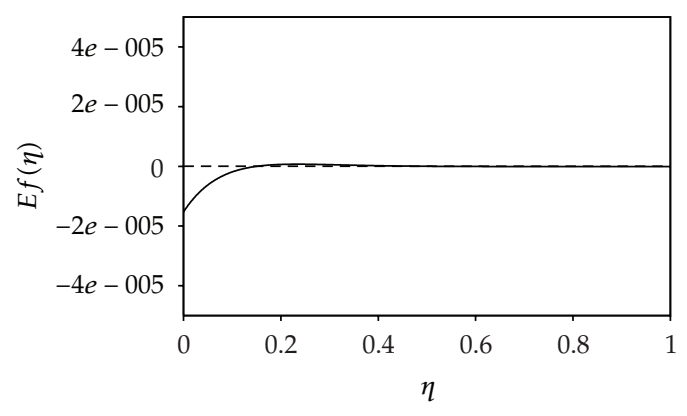

(a)

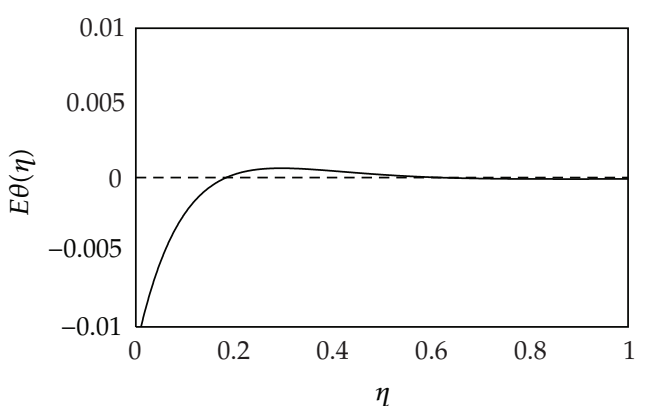

(b)

Figure 2: The residual errors for the solution using 15th-order HAM approximation for the case of $S=1.4$, $\operatorname{Pr}=1, M=1, N_{R}=1$, and $B^{*}=0.05$.

In order to assess the accuracy of the numerical method, Table 1 compares our present results with those of previous investigations. We can claim that our numerical results are in excellent agreement with those of $[4,14,24]$ under some limiting cases.

In Table 2, a good agreement was found in comparisons of values of free surface temperature $\theta(1)$ between [24] and present work.

The effects of the radiation parameter $N_{R}$ on the film thickness $\beta$, surface shear stress $f^{\prime \prime}(0)$, free surface temperature $\theta(1)$, and heat flux $-\theta^{\prime}(0)$ at a specific thermocapillarity 
Table 1: Values of dimensionless film thickness $\beta$ and skin friction $f^{\prime \prime}(0)$ when $\operatorname{Pr}=1, M=0, N_{R}=0$, $\hbar_{f}=-0.6$, and $\hbar_{\theta}=-0.4$ for different values of unsteadiness parameter $S$. The value of $f^{\prime \prime}(0) / \beta$ in Wang [4] and the present work is the same as that of Abel et al. [14] despite different similarity transformations used.

\begin{tabular}{ccccccccc}
\hline & \multicolumn{2}{c}{ Wang [4] } & \multicolumn{2}{c}{ Abel et al. [14] } & \multicolumn{2}{c}{ Mahmoud [24] } & \multicolumn{2}{c}{ Present work } \\
$S$ & $\beta$ & $f^{\prime \prime}(0) / \beta$ & $\beta$ & $f^{\prime \prime}(0)$ & $\beta$ & $f^{\prime \prime}(0)$ & $\beta$ & $f^{\prime \prime}(0) / \beta$ \\
\hline 0.4 & 5.122490 & -1.307785 & 4.981455 & -1.134098 & - & - & 5.126821 & -1.040765 \\
0.6 & 3.131250 & -1.195155 & 3.131710 & -1.195128 & - & - & 3.131668 & -1.193654 \\
0.8 & 2.151990 & -1.245795 & 2.151990 & -1.245805 & 2.1519950 & -1.245810 & 2.151994 & -1.245793 \\
1.0 & 1.543617 & -1.277762 & 1.543617 & -1.277769 & - & - & 1.543616 & -1.277768 \\
1.2 & 1.127780 & -1.279177 & 1.127780 & -1.279171 & 1.1277815 & -1.279170 & 1.127780 & -1.279172 \\
1.4 & 0.821032 & -1.233549 & 0.821033 & -1.233545 & - & - & 0.821032 & -1.233549 \\
1.6 & 0.567173 & -1.114937 & 0.576176 & -1.114941 & - & - & 0.576173 & -1.114937 \\
1.8 & 0.356389 & -0.867414 & 0.356390 & -0.867416 & - & - & 0.356389 & -0.867414 \\
\hline
\end{tabular}

Table 2: Values of free surface temperature $\theta(1)$ using 10th-order HAM approximation for several values of $M, N_{R}$ with $\operatorname{Pr}=0.1, B^{*}=0$, and $S=1.2$.

\begin{tabular}{lccc}
\hline$M$ & $N_{R}$ & & $\theta(1)$ \\
& & Mahmoud [24] & Present work \\
\hline 0 & 0.2 & 0.86681 & 0.866844 \\
0.1 & 0.2 & 0.85184 & 0.854257 \\
0.5 & 0.2 & 0.78815 & 0.810103 \\
0.1 & 0 & 0.82693 & 0.829606 \\
0.1 & 0.2 & 0.85184 & 0.854257 \\
0.1 & 1.0 & 0.90594 & 0.907657 \\
\hline
\end{tabular}

number $M$ are shown in Table 3. It is concluded that the film thickness and the free surface temperature increase as the radiation parameter increases and consequently the surface shear stress and dimensionless heat flux decrease.

Table 4 shows the effects of thermocapillarity number on the film thickness $\beta$, surface shear stress $f^{\prime \prime}(0)$, free surface temperature $\theta(1)$, and heat flux $-\theta^{\prime}(0)$. We agree with [24] that the thermocapillarity parameter has the effect of enhancing the velocity and the local Nusselt number while decreasing the temperature and the surface shear stress.

The heat absorption sink $\left(B^{*}<0\right)$ leads to a decrease in the thermal boundary layer whereas the boundary layer thickness increases with increase in $B^{*}$. These effects can be observed in Table 5. As the temperature-dependent parameter $B^{*}$ increases for both cases (heat source or heat sink), the free surface temperature increases while the surface shear stress and wall heat flux decrease.

The effects of the different values of radiation parameter $N_{R}$ on the temperature profiles are depicted in Figure 3. By fixing $M=1, S=1.4, \operatorname{Pr}=1$, and $B^{*}=0.05$, the thin film flow swings from slight deceleration to higher velocity while the temperature increases as $N_{R}$ increases.

Thermocapillarity produced an outward flow along the free surface. Figure 4 shows that by increasing the value of the thermocapillarity number, the velocity decreases until it arrives at a minimum and then increases to its free surface value while the temperature consistently cools down. 


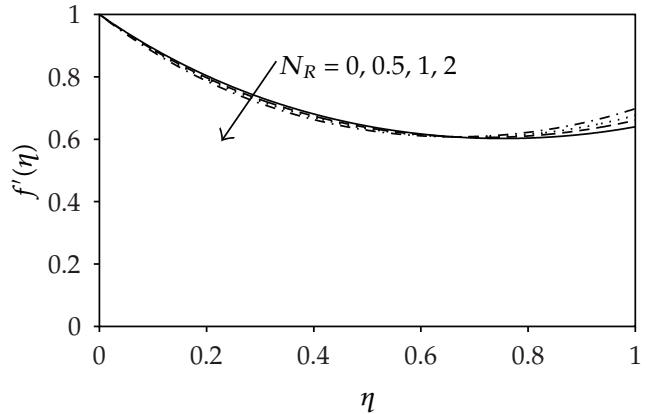

(a)

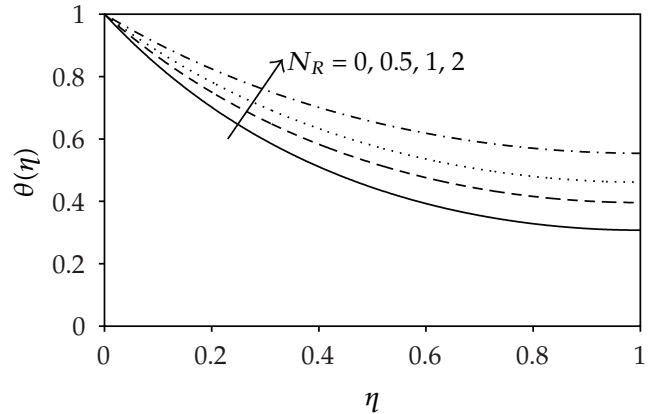

(b)

Figure 3: Effects of the radiation parameter $N_{R}$ on (a) $f^{\prime}(\eta)$ and (b) $\theta(\eta)$ using 10th-order HAM approximation for the case of $\hbar_{f}=-0.6, \hbar_{\theta}=-0.8, S=1.4, \operatorname{Pr}=1, M=1$, and $B^{*}=0.05$.

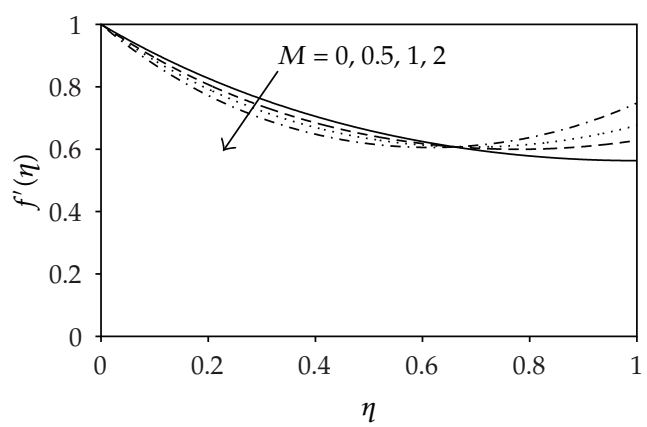

(a)

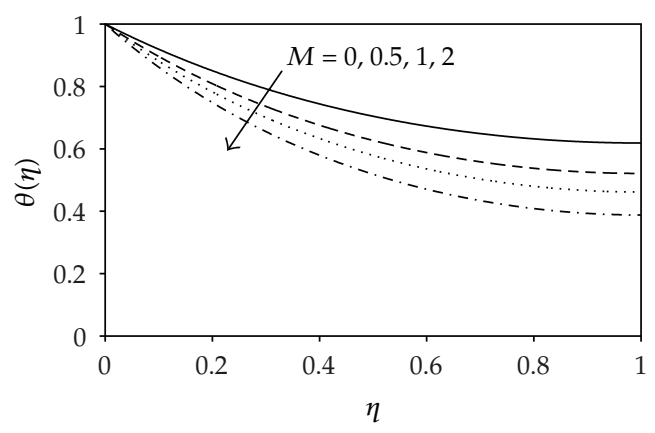

(b)

Figure 4: Effects of $M$ on (a) $f^{\prime}(\eta)$ and (b) $\theta(\eta)$ using 10th-order HAM approximation for the case of $\hbar_{f}=-0.6, \hbar_{\theta}=-0.8, S=1.4, N_{R}=1, \operatorname{Pr}=1$, and $B^{*}=0.05$.

Table 3: Variations of $\gamma(\eta), \beta, f^{\prime \prime}(0), \theta(1)$, and $-\theta^{\prime}(0)$ using 10th-order HAM approximation when $\operatorname{Pr}=1$, $M=1, B^{*}=0.05, S=1.4$, and $N_{R}$ is varied.

\begin{tabular}{lccccc}
\hline$N_{R}$ & $\gamma(\eta)$ & $\beta$ & $f^{\prime \prime}(0)$ & $\theta(1)$ & $-\theta^{\prime}(0)$ \\
\hline 0 & 1.008941 & 1.004460 & -1.201179 & 0.307714 & 1.841899 \\
0.5 & 1.103655 & 1.050550 & -1.252414 & 0.396179 & 1.512100 \\
1.0 & 1.173616 & 1.083335 & -1.289824 & 0.461740 & 1.299809 \\
1.5 & 1.227240 & 1.107809 & -1.318863 & 0.513259 & 1.147157 \\
2.0 & 1.270251 & 1.127054 & -1.341533 & 0.554268 & 1.031198 \\
\hline
\end{tabular}

Table 4: Variations of $\gamma(\eta), \beta, f^{\prime \prime}(0), \theta(1)$, and $-\theta^{\prime}(0)$ using 10th-order HAM approximation when $\operatorname{Pr}=1$, $N_{R}=1, B^{*}=0.05, S=1.4$, and $M$ is varied.

\begin{tabular}{lccccc}
\hline$M$ & $\gamma(\eta)$ & $\beta$ & $f^{\prime \prime}(0)$ & $\theta(1)$ & $-\theta^{\prime}(0)$ \\
\hline 0 & 0.674094 & 0.821032 & -1.012774 & 0.618739 & 0.873492 \\
0.5 & 0.969091 & 0.984424 & -1.172420 & 0.519700 & 1.140371 \\
1.0 & 1.173616 & 1.083335 & -1.299824 & 0.461740 & 1.299809 \\
1.5 & 1.350054 & 1.161918 & -1.382159 & 0.419534 & 1.425835 \\
2.0 & 1.499933 & 1.224717 & -1.459757 & 0.388086 & 1.524753 \\
\hline
\end{tabular}




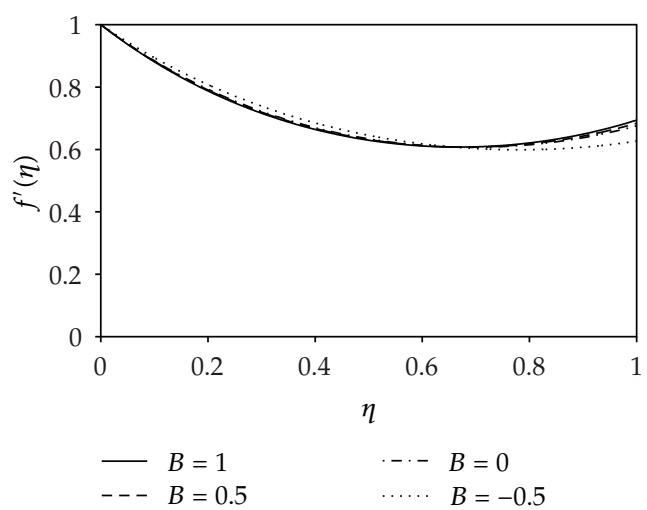

(a)

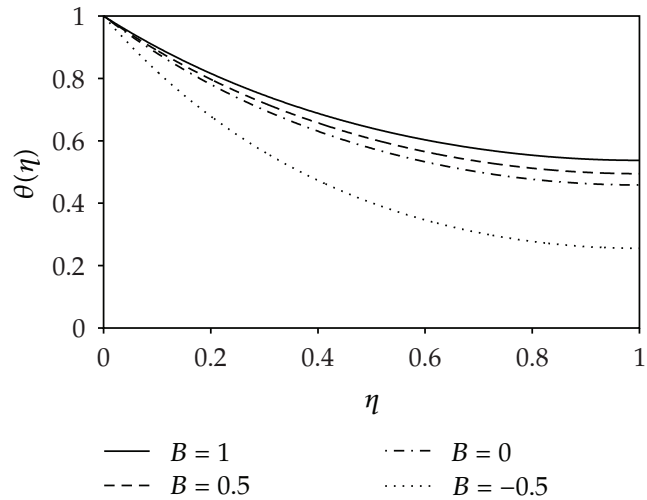

(b)

Figure 5: Effects of $B^{*}$ on (a) $f^{\prime}(\eta)$ and $(b) \theta(\eta)$ using 10th-order HAM approximation for the case of $\hbar_{f}=-0.6, \hbar_{\theta}=-0.8, S=1.4, M=1.0, N_{R}=1$, and $\operatorname{Pr}=1$.

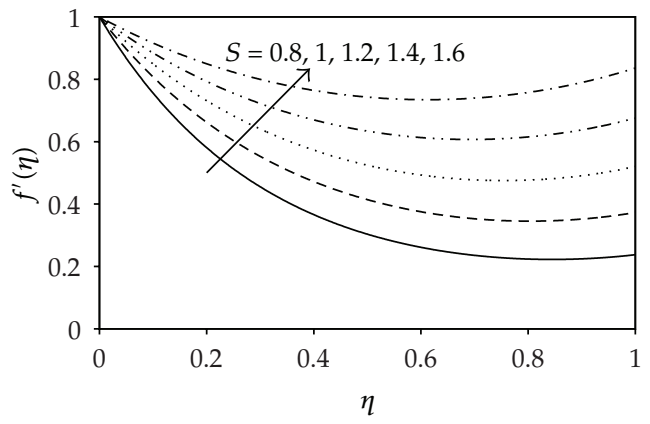

(a)

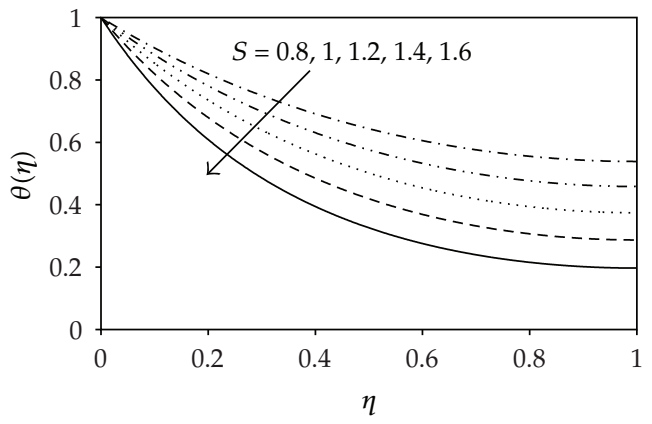

(b)

Figure 6: Effects of $S$ on (a) $f^{\prime}(\eta)$ and (b) $\theta(\eta)$ using 10th-order HAM approximation for the case of $\hbar_{f}=$ $-0.6, \hbar_{\theta}=-0.8, M=1.0, N_{R}=1, \operatorname{Pr}=1$, and $B^{*}=0.05$.

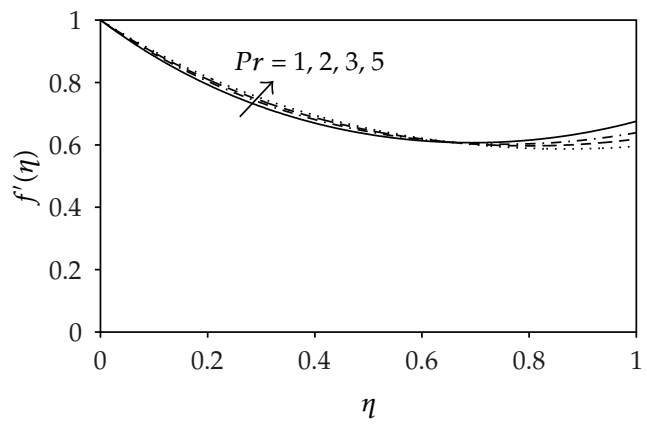

(a)

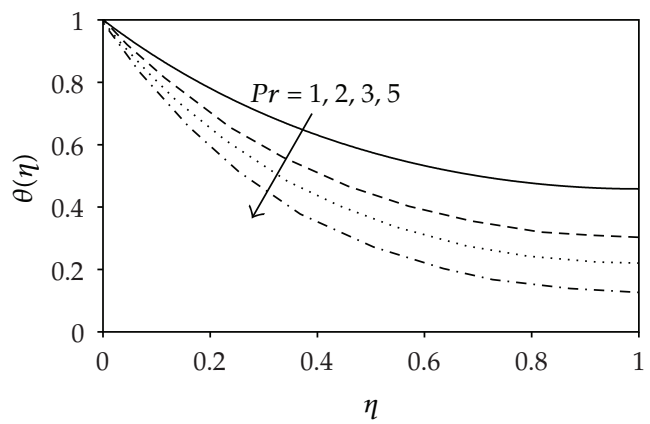

(b)

Figure 7: Effects of $\operatorname{Pr}$ on (a) $f^{\prime}(\eta)$ and (b) $\theta(\eta)$ using 10th-order HAM approximation for the case of $\hbar_{f}=-0.6, \hbar_{\theta}=-0.8, M=1.0, N_{R}=1, S=1.4$, and $B^{*}=0.05$. 
Table 5: Variations of $\gamma(\eta), \beta, f^{\prime \prime}(0), \theta(1)$, and $-\theta^{\prime}(0)$ using 10th-order HAM approximation when $\operatorname{Pr}=1$, $N_{R}=1, M=1, S=1.4$, and $B^{*}$ is varied.

\begin{tabular}{lccccc}
\hline$B^{*}$ & $\gamma(\eta)$ & $\beta$ & $f^{\prime \prime}(0)$ & $\theta(1)$ & $-\theta^{\prime}(0)$ \\
\hline-1.0 & 1.108464 & 1.052836 & -1.254129 & 0.399346 & 1.485058 \\
-0.5 & 1.137235 & 1.066412 & -1.270027 & 0.426965 & 1.401209 \\
0 & 1.170067 & 1.081696 & -1.287906 & 0.458354 & 1.309486 \\
0.5 & 1.206136 & 1.099152 & -1.308352 & 0.494630 & 1.207863 \\
1.0 & 1.253028 & 1.119387 & -1.332192 & 0.537395 & 1.093403 \\
\hline
\end{tabular}

Figure 5 shows that when the temperature-dependent parameter $B^{*}$ increases, the velocity decreases while the temperature increases. This is due to the fact that the internal heat source enhances or damps heat transport.

The unsteadiness parameter $S$ has the effect of increasing both velocity profiles and temperature distribution at a specific $N_{R}, B^{*}$, and $M$, as shown in Figure 6. The effects of Prandtl number Pr on temperature distribution is presented in Figure 7. As Pr increases, the velocity profile increases and the temperature distribution decreases. The higher the Prandtl number, the cooler the temperature of the fluid flow.

\section{Concluding Remarks}

The effects of thermal radiation and thermocapillarity in a thin liquid film on an unsteady stretching sheet with nonuniform heat source/sink was analyzed successfully by means of the homotopy analysis method (HAM). With the presence of internal heat generation/ absorption, the radiation parameter plays a significant role in controlling the temperature of the fluid flow by enhancing the temperature of fluid flow and decreasing the dimensionless heat flux. The thermocapillarity enhances the velocity and the dimensionless heat flux while reducing the temperature and the surface shear stress.

\section{References}

[1] C. Y. Wang, "Liquid film on an unsteady stretching surface," Quarterly of Applied Mathematics, vol. 48, no. 4, pp. 601-610, 1990.

[2] H. I. Andersson, J. B. Aarseth, and B. S. Dandapat, "Heat transfer in a liquid film on an unsteady stretching surface," International Journal of Heat and Mass Transfer, vol. 43, no. 1, pp. 69-74, 2000.

[3] I. C. Liu and H. I. Andersson, "Heat transfer in a liquid film on an unsteady stretching sheet," International Journal of Thermal Sciences, vol. 47, no. 6, pp. 766-772, 2008.

[4] C. Wang, "Analytic solutions for a liquid film on an unsteady stretching surface," Heat and Mass Transfer, vol. 42, no. 8, pp. 759-766, 2006.

[5] H. I. Andersson, J. B. Aarseth, N. Braud, and B. S. Dandapat, "Flow of a power-law fluid film on an unsteady stretching surface," Journal of Non-Newtonian Fluid Mechanics, vol. 62, no. 1, pp. 1-8, 1996.

[6] C. H. Chen, "Heat transfer in a power-law fluid film over a unsteady stretching sheet," Heat and Mass Transfer, vol. 39, no. 8-9, pp. 791-796, 2003.

[7] C. H. Chen, "Effect of viscous dissipation on heat transfer in a non-Newtonian liquid film over an unsteady stretching sheet," Journal of Non-Newtonian Fluid Mechanics, vol. 135, no. 2-3, pp. 128-135, 2006.

[8] C. Wang and I. Pop, "Analysis of the flow of a power-law fluid film on an unsteady stretching surface by means of homotopy analysis method," Journal of Non-Newtonian Fluid Mechanics, vol. 138, no. 2-3, pp. 161-172, 2006.

[9] M. A. A. Mahmoud and A. M. Megahed, "MHD flow and heat transfer in a non-Newtonian liquid film over an unsteady stretching sheet with variable fluid properties," Canadian Journal of Physics, vol. 87, no. 10, pp. 1065-1071, 2009. 
[10] B. S. Dandapat, B. Santra, and H. I. Andersson, "Thermocapillarity in a liquid film on an unsteady stretching surface," International Journal of Heat and Mass Transfer, vol. 46, no. 16, pp. 3009-3015, 2003.

[11] B. S. Dandapat, B. Santra, and K. Vajravelu, "The effects of variable fluid properties and thermocapillarity on the flow of a thin film on an unsteady stretching sheet," International Journal of Heat and Mass Transfer, vol. 50, no. 5-6, pp. 991-996, 2007.

[12] C. H. Chen, "Marangoni effects on forced convection of power-law liquids in a thin film over a stretching surface," Physics Letters Section A, vol. 370, no. 1, pp. 51-57, 2007.

[13] N. F. M. Noor and I. Hashim, "Thermocapillarity and magnetic field effects in a thin liquid film on an unsteady stretching surface," International Journal of Heat and Mass Transfer, vol. 53, no. 9-10, pp. 20442051, 2010.

[14] M.S. Abel, N. Mahesha, and J. Tawade, "Heat transfer in a liquid film over an unsteady stretching surface with viscous dissipation in presence of external magnetic field," Applied Mathematical Modelling, vol. 33, no. 8, pp. 3430-3441, 2009.

[15] N. F. M. Noor, O. Abdulaziz, and I. Hashim, "MHD flow and heat transfer in a thin liquid film on an unsteady stretching sheet by the homotopy analysis method," International Journal for Numerical Methods in Fluids, vol. 63, no. 3, pp. 357-373, 2010.

[16] S. Liao, Beyond Perturbation: Introduction to the Homotopy Analysis Method, vol. 2 of CRC Series: Modern Mechanics and Mathematics, Chapman \& Hall/CRC, Boca Raton, Fla, USA, 2004.

[17] S. Abbasbandy, "Homotopy analysis method for the Kawahara equation," Nonlinear Analysis. Real World Applications, vol. 11, no. 1, pp. 307-312, 2010.

[18] O. Abdulaziz, N. F. M. Noor, and I. Hashim, "Homotopy analysis method for fully developed MHD micropolar fluid flow between vertical porous plates," International Journal for Numerical Methods in Engineering, vol. 78, no. 7, pp. 817-827, 2009.

[19] O. Abdulaziz and I. Hashim, "Fully developed free convection heat and mass transfer of a micropolar fluid between porous vertical plates," Numerical Heat Transfer Part A, vol. 55, no. 3, pp. 270-288, 2009.

[20] T. Hayat, T. Javed, and Z. Abbas, "MHD flow of a micropolar fluid near a stagnation-point towards a non-linear stretching surface," Nonlinear Analysis. Real World Applications, vol. 10, no. 3, pp. 1514-1526, 2009.

[21] T. Hayat, M. Mustafa, and S. Asghar, "Unsteady flow with heat and mass transfer of a third grade fluid over a stretching surface in the presence of chemical reaction," Nonlinear Analysis. Real World Applications, vol. 11, no. 4, pp. 3186-3197, 2010.

[22] N. Kousar and S. Liao, "Unsteady non-similarity boundary-layer flows caused by an impulsively stretching flat sheet," Nonlinear Analysis. Real World Applications, vol. 12, no. 1, pp. 333-342, 2011.

[23] M. Abd El-Aziz, "Radiation effect on the flow and heat transfer over an unsteady stretching sheet," International Communications in Heat and Mass Transfer, vol. 36, no. 5, pp. 521-524, 2009.

[24] M. A. A. Mahmoud, "Thermal radiation effects on the flow and heat transfer in a liquid film on an unsteady stretching sheet," International Journal for Numerical Methods in Fluids, vol. 67, no. 11, pp. 1692-1701, 2011.

[25] S. Liao, "Notes on the homotopy analysis method: some definitions and theorems," Communications in Nonlinear Science and Numerical Simulation, vol. 14, no. 4, pp. 983-997, 2009. 


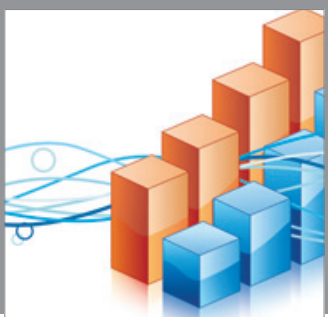

Advances in

Operations Research

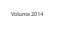

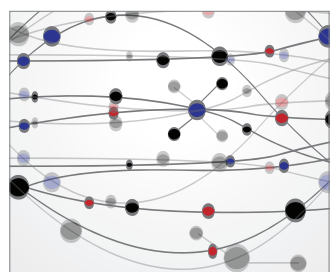

\section{The Scientific} World Journal
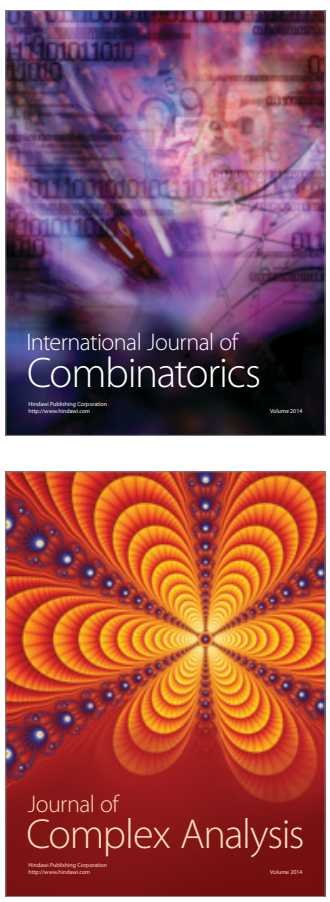

International Journal of

Mathematics and

Mathematical

Sciences
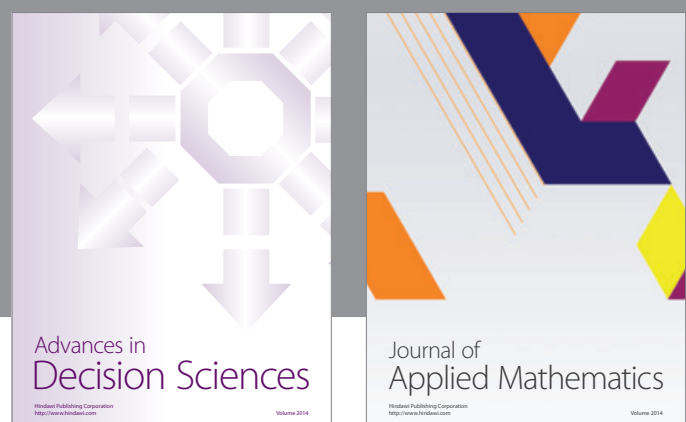

Journal of

Applied Mathematics
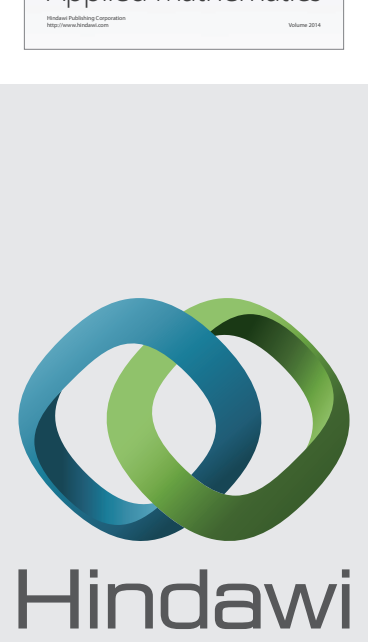

Submit your manuscripts at http://www.hindawi.com
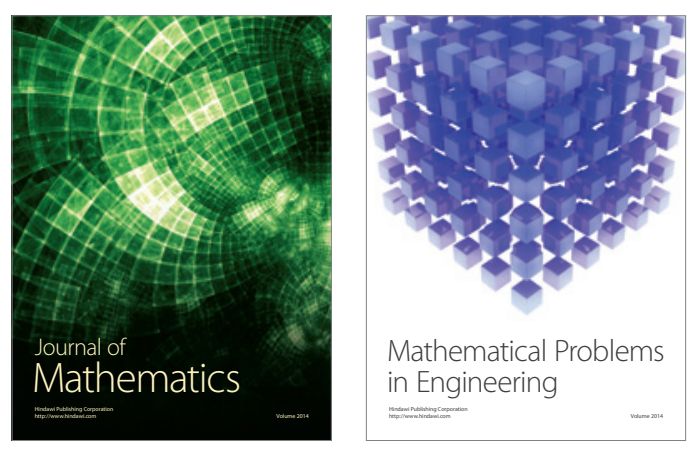

Mathematical Problems in Engineering
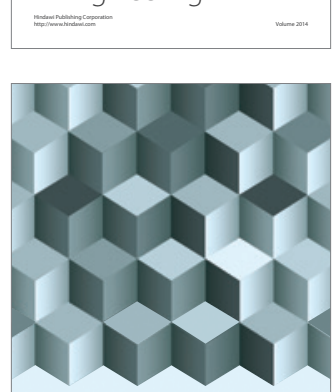

Journal of

Function Spaces
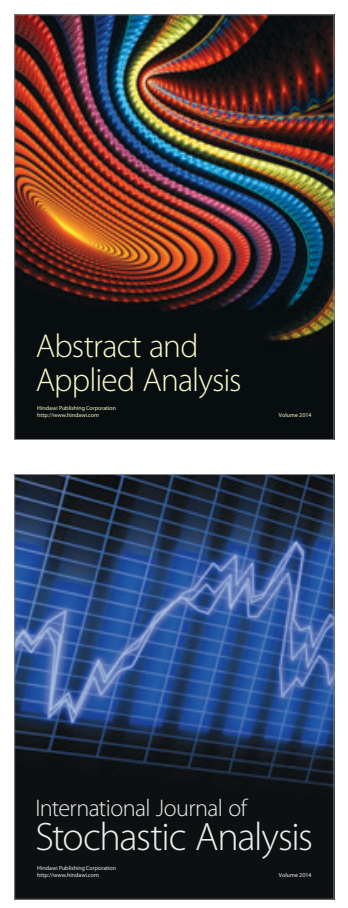

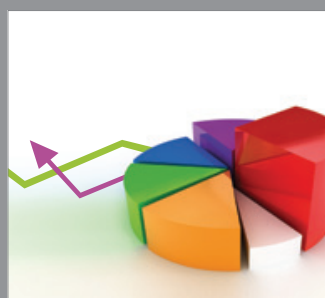

ournal of

Probability and Statistics

Promensencen
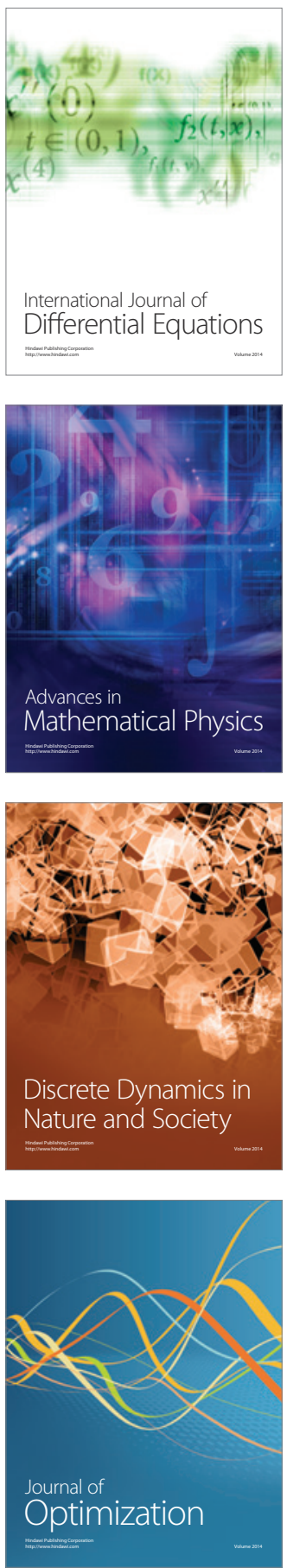